\title{
Autonomously Distributed Control of Electric Vehicle Chargers for Grid Services
}

Sevdari, Kristian; Calearo, Lisa; Striani, Simone; Andersen, Peter Bach; Marinelli, Mattia; Rønnow, Lauge

Published in:

Proceedings of ISGT Europe 2021

Link to article, DOI:

10.1109/ISGTEurope52324.2021.9640132

Publication date:

2021

Document Version

Peer reviewed version

Link back to DTU Orbit

Citation (APA):

Sevdari, K., Calearo, L., Striani, S., Andersen, P. B., Marinelli, M., \& Rønnow, L. (2021). Autonomously Distributed Control of Electric Vehicle Chargers for Grid Services. In Proceedings of ISGT Europe 2021 IEEE. https://doi.org/10.1109/ISGTEurope52324.2021.9640132

\section{General rights}

Copyright and moral rights for the publications made accessible in the public portal are retained by the authors and/or other copyright owners and it is a condition of accessing publications that users recognise and abide by the legal requirements associated with these rights.

- Users may download and print one copy of any publication from the public portal for the purpose of private study or research.

- You may not further distribute the material or use it for any profit-making activity or commercial gain

- You may freely distribute the URL identifying the publication in the public portal 


\section{Autonomously Distributed Control of Electric Vehicle Chargers for Grid Services}

\author{
Kristian Sevdari, Lisa Calearo, Simone Striani, \\ Peter Bach Andersen, Mattia Marinelli \\ Department of Electrical Engineering \\ Technical University of Denmark (DTU) \\ Roskilde, Denmark \\ \{krisse; lica; sistri; pba; matm\}@elektro.dtu.dk
}

\author{
Lauge Rønnow \\ Circle Consult Research and Development \\ Circle Consult \\ Nærum, Denmark \\ lr@circleconsult.dk
}

\begin{abstract}
As part of a sustainable power system, a synergy between electric mobility and renewable energy sources (RESs) can play a crucial role on mitigating the nature of RESs and defer costly grid upgrades via smart-charging. This paper presents a distributed autonomous control architecture for electric vehicle (EV) chargers and a clustering method for charging coordination. The architecture framework is detailed depending on the number of chargers and specific location properties. Moreover, the framework unveils the communication, measurement and power flow. The aforementioned approach aims at simplifying the overall charging experience for the EV owners while coupling it with a healthy grid behavior. The proposed control architecture is simulated on a prosumer case with two EVs. The performance of the controller is considerably affected by observability capabilities of current smart-meters. Faster measurement cycles of smartmeters can reduce the overshoot time span but not prevent it.
\end{abstract}

Index Terms-distributed control, electric vehicle, smartcharging, flexibility, prosumer

\section{INTRODUCTION}

As the society is increasing the electricity usage from RESs for its daily needs, the electrical grid experiences the consequences of this transformation. The goal for a sustainable power system showcases a paradigm shift, from generation to demand side control. Based on sustainable grid constraints, ramping flexibility and congestion management are some of the challenges for the future of the grid. To tackle these challenges enabling technologies like utility-scale, behind the meter batteries and EV smart charging are seen as a solution. The common characteristic is the ability to control the load and help demand match supply. Moreover, rapid EV deployment reflects a power delivery that, if left uncontrolled, can result in a concurrent consumption that can potentially overload the grid [1]. Another aspect is the requirement for continuous large residual generation ramping flexibility ( $\mathrm{MW} / \mathrm{min}$ ), which increase the costs for running the grid [2]. In this regard unidirectional smart-charging (V1G) becomes crucial for the future reinforcement of the grid, as it unleashes flexibility from EVs to help the grid accommodate a larger energy consumption [3]. Ref. [4] assesses flexibility, which comes as a shift or stretch on time of the charging process and adjustable power consumption. By adjusting charging current, the smart mechanism could further: reduce stress on the grid operation [5]; avoid or delay costly grid upgrades [6]; improve power quality [7]; minimize losses on distribution grid [8]; follow demand response programs [9]; make charging cheaper [10].

\section{A. Control architecture state-of-the-art}

Smart-chargers as a distributed infrastructure are the meeting point of the energy sector which incorporates the physical grid components and electricity market with the transport sector. Control and coordination of such infrastructure is achieved via centralized, decentralized, or distributed control architectures. Based on a thorough cover of the pros and cons of each method from [11], [12], below it is first compared the centralized versus the decentralized approaches, and afterwards the distributed control, which is also the most promising one towards distributed energy resource (DER) coordination. With the centralized approach the central intelligence, named cloud aggregator, controls all the EVs charging. While with the decentralized technique the intelligence, named virtual aggregator (VA), resides on each charger. Here, even though each charger autonomously runs its controlling actions, decisions of each controller can be influenced by price or a reference control signal from the aggregator, user or utility. The centralized approach is investigated for example in [13], where the aggregated power profile of EVs tracks a reference power resulting from electricity markets. However, the centralized control is quite vulnerable to the VA malfunction, resulting on a need for a backup system. The single server error of centralized control would spread over all chargers. On the contrary, the decentralized control is less sensitive to errors, hence increasing robustness of the system. For what concerns the optimization algorithms, those are easier to be implemented on centralized control, due to the system wide observation, compared to the lack of grid visibility experienced on decentralized control. Ref. [14] compares a charger control on local (decentralized) versus centralized grid measurements. The lack of visibility on local control case, resulted in a slightly lower efficiency compared to the centralized case. In addition, avalanche effects caused by price synchronizations, which is a common challenge for both architectures, should be carefully handled by the controller. In terms of communication protocol, the difference between centralized and decentralized VA control is the two-way (server-clients) versus one-way communication path (Fig. 1). The centralized 
architecture has a heavy operation in terms of communication and computation when it is scaled-up. On the contrary, the decentralized architecture requires less communication and computation capabilities [15], [16] and diverts data privacy challenges. Moreover, the one-way communication has the potential of implementing plug \& play protocols and simplify user interaction [17].

The third approach, distributed control, combines the benefits of centralized and decentralized control. It grows from decentralized control and tackles decentralized lack of visibility and control algorithms integration by introducing a vertical connection with the cloud aggregator [11]. Additionally, it can manifest a control hierarchy, which aligns with the grid physical structure by simplifying and distributing the control objective.

\section{B. Main contributions and charger design}

Taking as reference the above mentioned distributed control benefits, this paper proposes a distributed autonomous charging control architecture for providing grid services. To achieve the desired control for the charger, two designs were considered: (i) first, a VA and a dumb charger device separated, where a single VA can control multiple dumb chargers; (ii) second, VA is included in each charger, making it a single device. Here, both charger designs qualify as part of on-board EV charger. From IEC 61851 standard [18], it can adjust its current from 6 to 16 Amps (maximum $11.09 \mathrm{~kW}$ ) with $1 \mathrm{Amp}$ discrete modulation, as shown at [19]. Currently, the state-ofthe-art of smart-charger technology employs the first design, [20] as relevant representatives. A recent initiative of using the second design is followed by Zaptec [21], however with a centralized control approach. On our research the most important aspect of the charger and VA operation is their ability to run autonomously at the largest possible extend. Since the first design is vulnerable of the VA being compromised and losing control of a set of chargers, the second design moved forward and is presented in this manuscript. To tackle the above-mentioned shortcomings of the first charger design, the second design has three pieces:

1) Measurement component: the local grid parameters.

2) Virtual aggregator component: the charger intelligence.

3) Charging component: the protection and charging port. The rest of the paper is structured as follows. Section II outlines a clustering method for autonomous EV chargers, and presents the autonomous control architecture together with the simulation model. Section III reports the results from the study case and section IV concludes the manuscript.

\section{Methodology}

The distributed control approach for a scaled-up charger deployment requires to coordinate different actors, namely: market, transmission system operator (TSO), distribution system operator (DSO), cloud aggregator, zonal VA and user. For this reason, the proposed clustering approach and control framework facilitate the interaction between actors.

\section{A. Clustering method}

Fig.1 and Table I provide and visualize the clustering method characteristics. Zones cluster chargers based on the number of the EV chargers located behind the same meter and together with the user form the core functioning environment between two actors. Each zone can perform its decisionmaking for charging operations autonomously, because the cloud aggregator is not vital and does not conflict with local operation goal. However, it helps to perform cross-actor interactions. A short description of each zone follows.

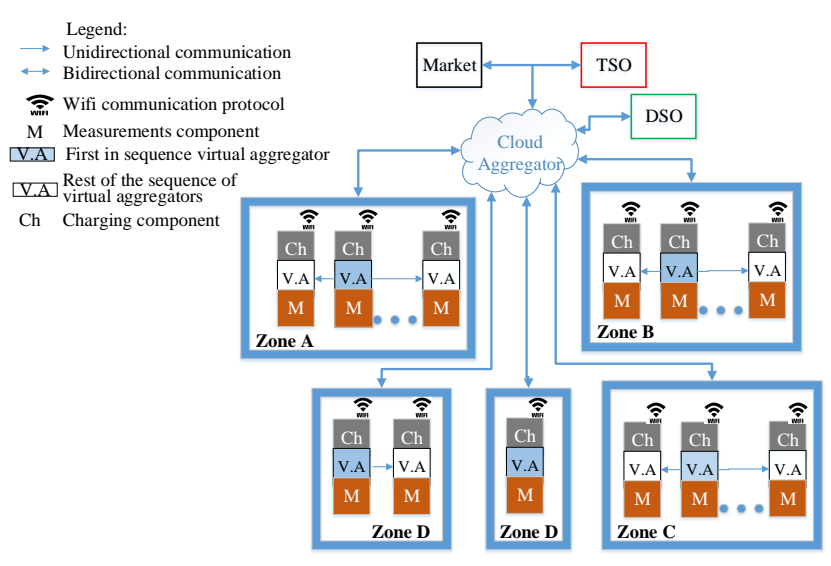

Fig. 1: Visualization of clustering method and communication paths.

TABLE I: Characteristics of the clustering method.

\begin{tabular}{|c|c|c|c|c|c|c|}
\hline $\begin{array}{c}\text { Zone } \\
\text { type }\end{array}$ & $\begin{array}{c}\text { Load } \\
\text { size }\end{array}$ & $\begin{array}{c}\text { No. of } \\
\text { chargers }\end{array}$ & Independent & $\begin{array}{c}\text { Coupled with } \\
\text { local load }\end{array}$ & $\begin{array}{c}\text { Type of } \\
\text { local load }\end{array}$ & $\begin{array}{c}\text { Coupled with } \\
\text { zonal load }\end{array}$ \\
\hline A & High & $20+$ & Yes & Yes & $\begin{array}{c}\text { Industrial/ } \\
\text { Commercial }\end{array}$ & Yes/No \\
\hline B & Medium & $3-20$ & Yes & Yes & $\begin{array}{c}\text { Industrial// } \\
\text { Commercial/ } \\
\text { Residential }\end{array}$ & Yes/No \\
\hline C & Low-Medium & $3-20$ & Yes & No & - & Yes/No \\
\hline D & Low & $1-2$ & Yes & Yes & $\begin{array}{c}\text { Commercial/l } \\
\text { Residential }\end{array}$ & Yes/No \\
\hline
\end{tabular}

Zone A represents a large-size charging infrastructure. This is the case of a parking lot of a stadium, airport, university campus, charging forecourt, etc.. Zone B describes a mediumsize charging infrastructure. This can be the parking lot of a school, theater, library, government building etc.. Zone $\mathrm{C}$ characterizes a small or medium-size charging infrastructure, dedicated only to charging EVs. Some examples are: public/private parking lots, curbside or garage charging infrastructure. Zone D employs a smaller number of chargers. This zone reflects charging infrastructure of residential houses. In this article, zone D is the case study and it is further explained in Section II-B. Inside the zones, each unit incorporate the same hardware and software, but they have a numbering sequence to specify the order. If the first VA suffers a malfunction, then the second VA replaces its role. By doing so, the leading "token" can be attached to each unit when needed, and provide a robust operation for the zone. 


\section{B. Control framework}

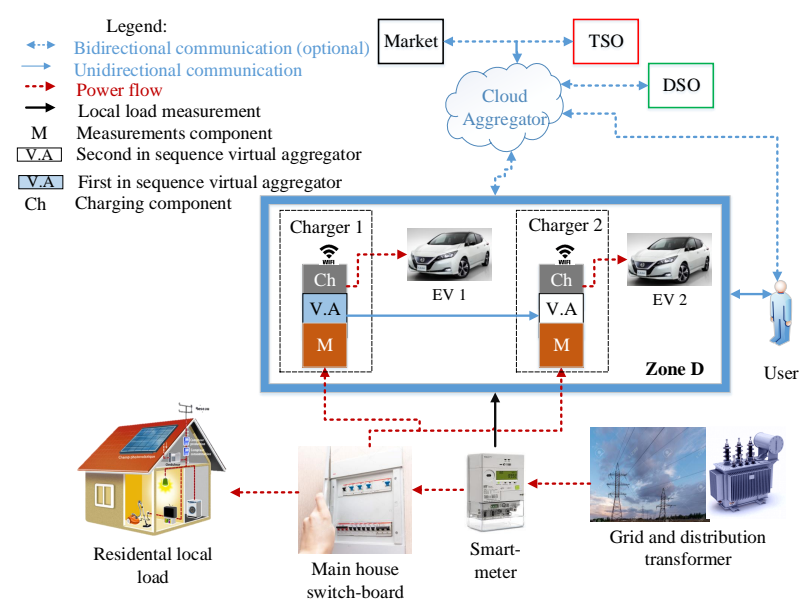

Fig. 2: Autonomous EV charger control architecture for zone D.

Zone $\mathrm{D}$ is the typical representation of coupling the consumer or prosumer with the EV charging needs and utility signals. Fig. 2 shows the power flow and information path for the charging operation. The first VA takes care of running the operation in the zone and broadcasts its signal to the nearby VA. The charger takes input signals from the user, the smartmeter and the cloud aggregator. Based on these inputs, the charger decides a charging current for the EV. Depending on the needs and user decision, the charging operation can focus on self-efficiency, time of use tariffs and better utilization of DERs, like rooftop photovoltaic (PV) panels. Furthermore, through the cloud aggregator the charging operation can be part of a bigger picture, coordinated by utility, system operator or market needs. An example for this case is a loading threshold signal, set on the local distribution transformer by the grid operator, limiting the charging current of nearby chargers connected with the transformer. Hence, based on the input signals, different charging modes are offered to the user without compromising his commodity. To summarize, each of these operation modes rely on not harming the grid, as it incentives a behind the meter responsible behavior from the EV user via economic benefits (such as lowering electricity bills, deferring grid upgrades and utilizing local generation). It is relevant to observe that cloud aggregator information regarding market, TSO and DSO controlling actions do not prevent running the local operation, thus in this paper early results regarding local operation are presented.

\section{Simulation model}

The simulation model representing zone D control framework is displayed in Fig. 3. From left to right, the point of common coupling (PCC) represents the power flow at the smart-meter. The smart-meter measures the active power and transmits this data to the leading VA with a certain measurement delay $\left(e^{-s \tau 1}\right)$ equal to 1 second. In addition, the meter has a 10 seconds measurement cycle [22]. This limitation affects visibility of fast dynamics in the smartmeter power-flow. Next, VA1 represents the VA in charge, whereas VA2 is the backup option. The VA1 calculates an available power and broadcasts it with a VA processing delay (0.5 second), marked as $e^{-s \tau 2}$. The broadcast of available charging power from VA1 is intended to happen every second to serve even as a time measurement for the backup operation, hence the broadcast delay $e^{-s \tau 4}$. If the following VA does not receive a signal after 3 broadcast cycles (equal to 3 seconds), it will take over the operation. In addition, the charger employes a proportional integral derivative (PID) controller that chooses between 3 sets of PID coefficients based on a priority designed according to state-of-charge (SoC) and user availability (Fig. 3 ). Here, the initial SoC is required to be manually provided by the user, together with battery capacity $[\mathrm{kWh}]$ and expected departure time. In Fig. 3, $P D_{\mathrm{i}}$ is the priority coefficient (with a range from 0 to 100), Userbehavior ${ }_{i}$ is the available hours (value from 1 to 24 ) and SoCout $\mathrm{i}_{\mathrm{i}}$ is the state-of-charge during charging for $\mathrm{EV} i$. Depending on the values of $\mathrm{PD}_{\mathrm{i}}$ there is: (i) low-priority (LP) when $\mathrm{PD}_{i}<33$, (ii) medium-priority (MP) when $33>\mathrm{PD}_{i}<67$ and (iii) high-priority (HP) when $67>\mathrm{PD}_{i}$. The last part of the model is the EV part. According to [18], there is a certain delay $\left(e^{-s \tau 3}\right.$, on average 2 seconds) from the moment the EV receives the signal to start charging and when it reacts to that signal.

To summarize, the control objective is to modulate the output power of each charger, in order to follow a reference demand after receiving the measured demand from the PCC.

\section{RESULTS}

In this paper early proof of concept results are presented. The simulations are performed for three-phase environment and cover two cases:

1) Case 1: Constant house load (HL) (1.5 kW) and one charger, which goes live with different priorities.

2) Case 2: Constant HL (1.5 kW), first and second charger going live in sequence with three-scenarios on charger priority and charging on local PV production.

\section{A. Case 1}

Fig.4 displays a 50 seconds simulation of the Case 1. The charger goes live and starts charging after 10 seconds. Here, it can be noted that once it starts charging, the reference power is activated. The reference demand is $14 \mathrm{~kW}$, reflecting a static maximum desired consumption from the grid. While the 35 Amps grid supply connection allows for a maximum of $24 \mathrm{~kW}$ power draw. Moreover, based on the user priority, different charging curves are experienced. The high, medium and low priority need around 16, 32 and 42 seconds each, to reach full charging power $(11.09 \mathrm{~kW})$, if there is enough available power. However, due to the smart-meter measurement cycle, the system experiences a lack of measurement visibility at PCC. For that reason, Fig.5 displays the actual and the measured power flow at PCC. Depending on the cycle range, the quality of the control will deteriorate (larger cycle) or improve (smaller cycle). Besides, the available power calculated and broadcasted 


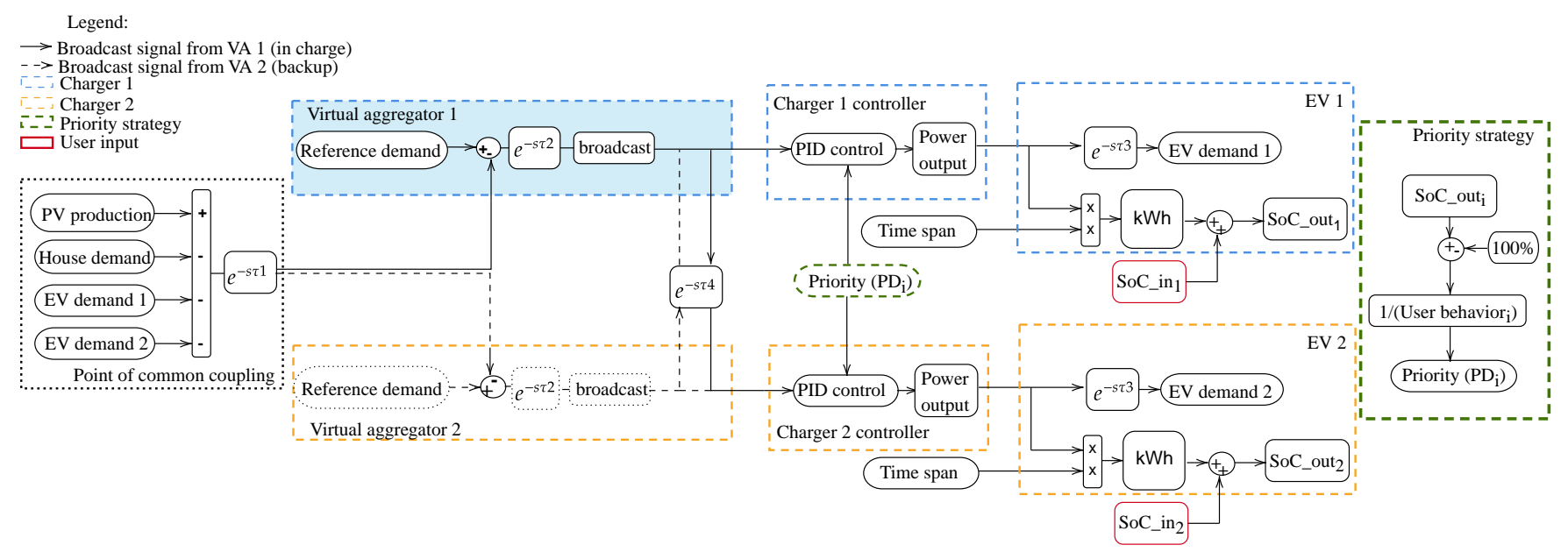

Fig. 3: Simplified simulation model for zone D.

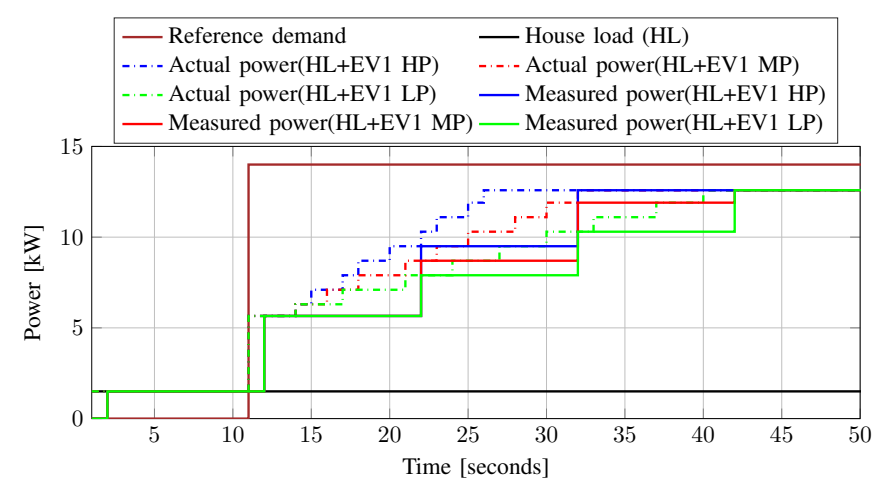

Fig. 4: Case 1: reference demand, house load, actual and measured power at PCC with charger being on high-priority (HP), medium-priority (MP) and low-priority (LP). Actual and measured power differ due to 10 seconds measurement cycle and 1 second delay.

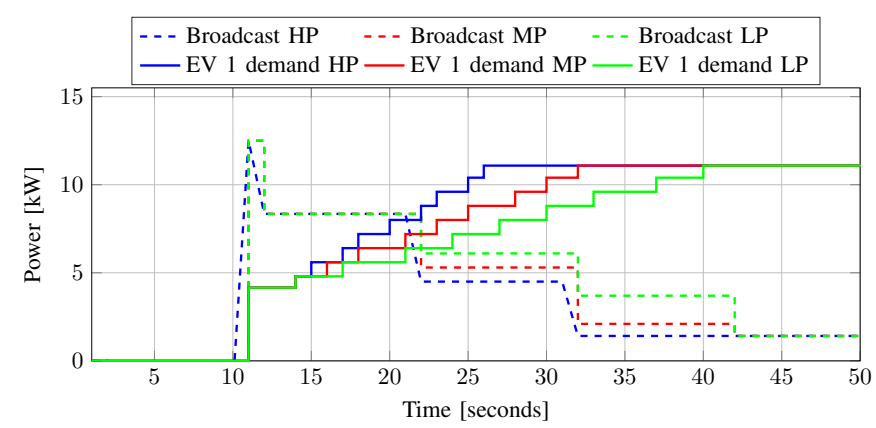

Fig. 5: Case 1: VA broadcast and EV charging power with high-priority (HP) medium-priority (MP) and low-priority (LP).

by VA1 can be distinguished in Fig. 5, together with the charging power delivered for each priority case. The broadcasted power (BP) from Fig.5 and the measured power curves for each scenario from Fig.4, are a mirror of each other. Moreover, after charging power saturates, the VA1 BP displays $1.415 \mathrm{~kW}(14-1.5-11.09=1.415 \mathrm{~kW})$ power availability. To conclude, the overall system delay counts for 14 seconds, where 10 seconds come from measurement cycle and 4 seconds from the remaining system delays (Fig.4).

\section{B. Case 2}

The second case introduces a second charger and a local PV generation (Fig.6). The goal for this case is to charge only with PV generation and for that reason the reference demand is equal to $0 \mathrm{~kW}$. This approach prevents a power flow from the grid. Here, three scenarios are considered: (1) EV1 and EV2 have HP; (2) EV1 has MP and EV2 has HP; (3) EV1 has LP and EV2 has HP. Furthermore, the actual consumption curves reflect the total consumption of the house plus first charger going live after 10 seconds and second charger after 50 seconds with different priorities. Besides, in Fig.6, PV power has negative value reflecting negative power flow (exporting to the grid) at PCC. In Fig.6, for all scenarios at 40-th second the first charger has saturated. The introduction of the second charger with an immediate power step of $4.15 \mathrm{~kW}(6 \mathrm{~A})$ tests the dynamic response of the controller. Additionally to cyclic measurements, the speed of the PID controller affects the quality of control and the reaction time, hence cannot prevent an overshoot from the reference demand, Fig.7. This is a physical constraint that should be carefully handled by the reference demand assigned to VA or a faster safety logic.

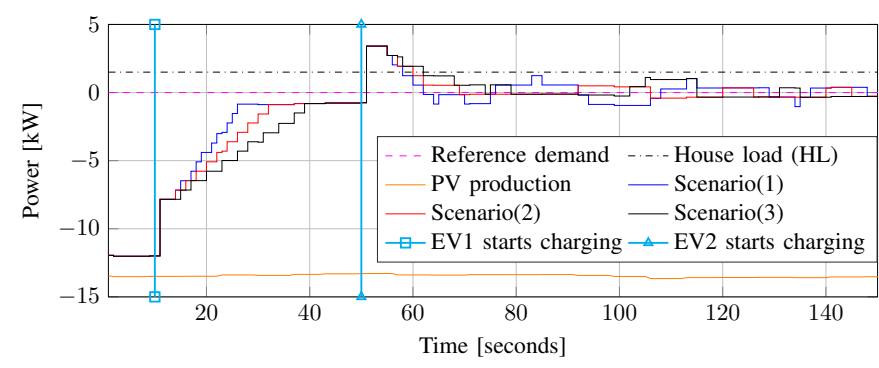

Fig. 6: Case 2: Actual power flow at PCC, scenarios (1),(2) and (3), with local generation and two chargers going live in sequence at 10-th and 50-th second, respectively. Negative power means export, positive means import 


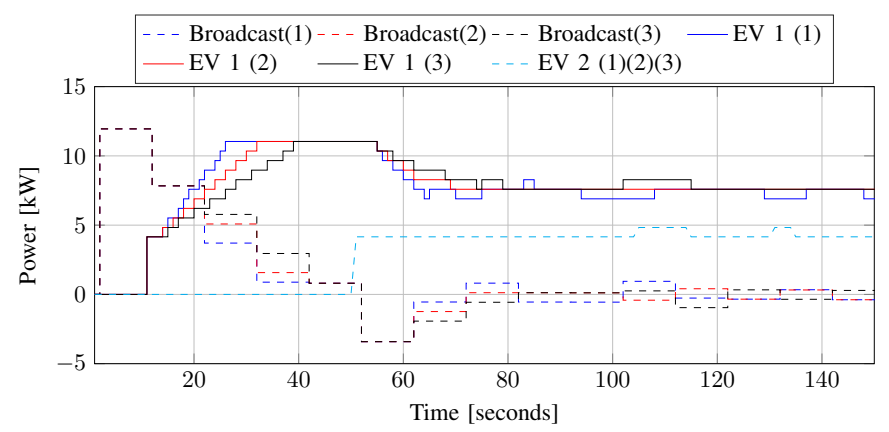

Fig. 7: Case 2: VA power broadcast and EVs charging power with first and second charger going live in sequence at 10-th and 50-th second, respectively. In addition, EVs follow three scenarios (1),(2) and (3).

Here, the overshoot is similar for all scenarios, equal to $3.412 \mathrm{~kW}$. However, the period staying above reference differs. Besides, it is emphasized, the higher the priority the faster the controlling action will compensate. Overshoot spans 14, 19 and 24 seconds for scenario (1),(2) and (3), respectively. Meanwhile, the time period of maximum overshoot stretches equally for all scenarios, 9 seconds. Here, 9 seconds is the worst case and 1 second is the best case, as it reflects the time between two measurements from the smart-meter, depending when the load step happens compared to smartmeter measurement. Moreover, Fig.7 displays the charging power occupied by each charger together with the broadcasted available power. In all scenarios, the second charger occupies the same minimum power $(4.15 \mathrm{~kW})$. While, the first charger is the one modulating its power to accommodate the second charger and follow the reference demand.

\section{CONCLUSION}

This paper introduced an autonomous EV charger control architecture with the goal of defining a distributed control architecture. Furthermore, the proposed clustering method facilitates the interaction between actors. The VA is included in each charger, characterizing the charger control with simplicity and scalability implementation. Moreover, the control framework is simulated on a prosumer case. Simulations displayed the quality of the control by evaluating the priority, speed, overshoot margin of the controller, and how it can follow the local PV generation. The overall system delays, the lack of measurement visibility and speed of the controller cannot prevent demand to overshoot the reference. However, the margin of allowed overshoot and implementation of back-up control in order to arrest overshoots will be further investigated on future work.

\section{ACKNOWLEDGEMENT}

The work in this paper is supported by the research projects ACDC (EUDP grant number: 64019-0541) and FUSE (EUDP grant number: 64020-1092).

\section{REFERENCES}

[1] L. Calearo, A. Thingvad, K. Suzuki, and M. Marinelli, "Grid loading due to ev charging profiles based on pseudo-real driving pattern and user behavior," IEEE Transactions on Transportation Electrification, vol. 5, pp. 683-694, 2019.
[2] ENTSO-E, "Ten-year network development plan : System dynamic and operational challenges," tech. rep., ENTSO-E, 2020.

[3] J. A. Peças Lopes, F. J. Soares, P. M. Almeida, and M. Moreira Da Silva, "Smart charging strategies for electric vehicles: Enhancing grid performance and maximizing the use of variable renewable energy resources," 24th International Battery, Hybrid and Fuel Cell Electric Vehicle Symposium and Exhibition 2009, EVS 24, vol. 4, pp. 2680-2690, 2009.

[4] K. Knezović, M. Marinelli, A. Zecchino, P. B. Andersen, and C. Traeholt, "Supporting involvement of electric vehicles in distribution grids: Lowering the barriers for a proactive integration," Energy, vol. 134, pp. 458-468, 2017.

[5] A. Schuller, J. Ilg, and C. Van Dinther, "Benchmarking electric vehicle charging control strategies," 2012 IEEE PES Innovative Smart Grid Technologies, ISGT 2012, 2012.

[6] C. Crozier, T. Morstyn, and M. McCulloch, "The opportunity for smart charging to mitigate the impact of electric vehicles on transmission and distribution systems," Applied Energy, vol. 268, no. March, p. 114973, 2020.

[7] L. Calearo, A. Thingvad, H. H. Ipsen, and M. Marinelli, "Economic Value and User Remuneration for EV Based Distribution Grid Services," Proceedings of 2019 IEEE PES Innovative Smart Grid Technologies Europe, ISGT-Europe 2019, 2019.

[8] E. Sortomme, M. M. Hindi, S. D. MacPherson, and S. S. Venkata, "Coordinated charging of plug-in hybrid electric vehicles to minimize distribution system losses," IEEE Transactions on Smart Grid, vol. 2, no. 1, pp. 198-205, 2011.

[9] M. H. Albadi and E. F. El-Saadany, "A summary of demand response in electricity markets," Electric Power Systems Research, vol. 78, no. 11, pp. 1989-1996, 2008.

[10] J. Dixon, P. B. Andersen, K. Bell, and C. Træholt, "On the ease of being green: An investigation of the inconvenience of electric vehicle charging," Applied Energy, vol. 258, no. November 2019, p. 114090 , 2020.

[11] X. Han, K. Heussen, O. Gehrke, H. W. Bindner, and B. Kroposki, "Taxonomy for Evaluation of Distributed Control Strategies for Distributed Energy Resources," IEEE Transactions on Smart Grid, vol. 9, no. 5, pp. 5185-5195, 2018.

[12] J. García-Villalobos, I. Zamora, J. I. San Martín, F. J. Asensio, an V. Aperribay, "Plug-in electric vehicles in electric distribution networks: A review of smart charging approaches," Renewable and Sustainable Energy Reviews, vol. 38, pp. 717-731, 2014.

[13] A. Di Giorgio, F. Liberati, and S. Canale, "IEC 61851 compliant electric vehicle charging control in smartgrids," 2013 21st Mediterranean Conference on Control and Automation, MED 2013 - Conference Proceedings, pp. 1329-1335, 2013.

[14] P. Richardson, D. Flynn, and A. Keane, "Local versus centralized charging strategies for electric vehicles in low voltage distribution systems," IEEE Transactions on Smart Grid, vol. 3, pp. 1020-1028, 2012.

[15] C. Ziras, A. M. Prostejovsky, H. W. Bindner, and M. Marinelli, "Decentralized and discretized control for storage systems offering primary frequency control,” Electric Power Systems Research, vol. 177, p. 106000,2019

[16] L. Gan, U. Topcu, and S. H. Low, "Optimal decentralized protocol for electric vehicle charging," IEEE Transactions on Power Systems, vol. 28 , no. 2, pp. 940-951, 2013.

[17] Y. Oki, T. Ogawa, and J. Kawaguchi, "Train power demand control using decentralized and parallel control scheme," IEEJ Journal of Industry Applications, vol. 6, no. 6, pp. 482-489, 2017.

[18] International Electrotechnical Commission, "IEC 61851." https:// webstore.iec.ch/publication/33644, 2017. Accessed: 26.02.2021.

[19] M. Marinelli, S. Martinenas, K. Knezović, and P. B. Andersen, "Validating a centralized approach to primary frequency control with seriesproduced electric vehicles," Journal of Energy Storage, vol. 7, pp. 63-73, 2016.

[20] L. Calearo, K. Sevdari, and M. Marinelli, "Status e-mobility DK," tech. rep., DTU - Technical University of Denmark, 2021.

[21] K. Sevdari, "Electric Vehicle Chargers Market Outlook," tech. rep., DTU - Technical University of Denmark, 2020.

[22] Aidon, "Han, Interface Deformation." https://www.nek.no/wp-content/ uploads/2018/11/Aidon-HAN-Interface-Description-v10A-ID-34331. pdf. Accessed: 26.02.2021. 